\title{
Development of a training package to increase the performance of radiographers in assessing screening mammograms
}

\begin{abstract}
Purpose: Due to a lack of any formal training in mammographic image interpretation for radiographers in Australia, a training package was developed specifically for radiographers who were to undertake a trial of screening mammogram assessment. Methods: A PowerPoint presentation was developed and included topics such as: how best to prepare to screen read, methods of checking all areas of the breast tissue, and examples of benign and malignant lesions. The training package was made available after completion of a pilot study, and pre-post testing was conducted on the same mammograms to determine the effectiveness of training. Results: There was an overall increase in sensitivity (73-78\%) with five of seven radiographers improving. The average specificity was relatively constant (71-70\%), with three radiographers showing improvement. Again, overall accuracy remained constant $(72 \%)$ with four radiographers improving, one remaining constant and two decreasing in accuracy. Conclusion: Guidelines for preparing and systematically checking mammogram images were helpful to most of the radiographers, with the training package helping to improve sensitivity and accuracy for the majority of participants.
\end{abstract}

S Moran

British Diploma of Diagnostic Radiography

Assoc Prof H Warren-Forward BSc (Hons), PhD

Medical Radiation Science, School of Health Sciences Faculty of Health

University of Newcastle Callaghan Campus New South Wales 2308, Australia.

Correspondence to Sheila.moran@uon.edu.au

Keywords: accuracy, improvement, mammograms, training.

\section{Introduction}

The number of women being screened through BreastScreen Australia programmes is steadily increasing ${ }^{1}$ and radiologist numbers are not maintaining pace with the aging Australian population, increased longevity and technological workload increases. ${ }^{2}$ This shortage of radiologists is more acute in the field of breast imaging, due to perceptions that it is not as stimulating, not as lucrative as other radiological modalities and carries a higher risk of litigation issues. ${ }^{3,4}$

BreastScreen Australia programmes undertake double reporting routinely. ${ }^{5}$ It has been documented that the more readers looking at the same images results in more cancers being detected; ${ }^{6,7}$ however this has to be balanced against increases in the recall and false-positive rates which are not cost-effective for BreastScreen programmes. Excessive false-positives may result in increased anxiety and has the potential to dissuade women from complying with regular rescreens. ${ }^{8}$

\section{Rationale}

The radiologist shortage was highlighted in 2004 in Newcastle, NSW when the BreastScreen New South Wales Hunter New England (BSNSW HNE) service closed for four months due to being unable to read screening mammograms or to undertake assessment clinics. This situation may have inconvenienced many women and caused some delays in resumption of regular attendances, which had the potential of delaying any cancer diagnoses. It is important that strategies are devised to prevent a reoccurrence in other BreastScreen services.

While radiographers are not formally trained to assess mammograms, they do examine mammogram images every day and provide the work-up images in assessment clinics. This experience could be put to use by training selected radiographers to become additional readers. This would potentially have a two-fold effect; extra readers (over and above the double reader policy) may increase the cancer detection within the programme $\mathrm{e}^{7,9}$ and, providing the radiographers maintained high standards, they could potentially provide continuity of service assessment if another shortage of radiologists seemed likely. The National Accreditation Standards allow for nonradiologist readers when the need arises. ${ }^{5}$

Radiographer reporting has been undertaken in countries other than Australia for two decades. Van den Biggelaar, et al. undertook a systematic review of literature and highlighted six studies focusing on the performance of radiographers interpreting mammograms between 1987 and 1996. ${ }^{10}$ All the studies were conducted in a screening setting and three measured the effect of training on the mammographers. The results showed a sensitivity range of $73-86 \%$ for the radiologists and $73-90 \%$ for the radiographers. Specificity for the radiologists was $81-95 \%$ and the mammographers achieved $64-91 \%$. The authors concluded that training programmes could improve radiographers' performance not only to increase cancer detection, but to identify benign lesions, which would increase specificity and keep recall rates low. ${ }^{11}$

In the UK Wivell, et al. documented a trial in 2002 by two radiographers who had been formally trained (Post-graduate Certificate in Image 
Table 1: Guide to assessment - adapted from the works of Tabar and Kopans. ${ }^{13,14}$

\begin{tabular}{|c|c|c|}
\hline & Action/question & Details \\
\hline 1 & Find it & $\begin{array}{l}\text { Perception of any abnormality (distortion, } \\
\text { asymmetry, skin change) }\end{array}$ \\
\hline 2 & Analyse it & $\begin{array}{l}\text { Is it real? Are normal structures overlapping? Is } \\
\text { it an artefact? }\end{array}$ \\
\hline 3 & Where is it? & Can it be located in 3D? \\
\hline 4 & What is it? & $\begin{array}{l}\text { Mass (circumscribed, stellate), distortion or } \\
\text { calcification }\end{array}$ \\
\hline 5 & What should be done? & $\begin{array}{l}\text { Has it been assessed previously? Categorise } \\
\text { result code }\end{array}$ \\
\hline
\end{tabular}

Table 3: Pre- and post-training measurements of the Pilot study images ( $^{*}$ indicates an increase in performance).

\begin{tabular}{llllllll} 
Readers & R1 & R2 & R3 & R4 & R5 & R6 & R7 \\
\hline $\begin{array}{l}\text { Sensitivity }(P=0.29) \\
\text { Pre-train }\end{array}$ & 61 & 72 & 61 & 67 & 89 & 78 & 83 \\
Post train & $73^{*}$ & $87^{*}$ & $67^{*}$ & 50 & $100^{*}$ & $88^{*}$ & 80 \\
Specificity $(P=0.76)$ & & & & & & & \\
Pre-train & 81 & 45 & 78 & 78 & 65 & 69 & 84 \\
Post train & 76 & $64^{*}$ & 55 & 62 & $74^{*}$ & $74^{*}$ & 83 \\
Accuracy $(P=0.91)$ & & & & & & & \\
Pre-train & 74 & 55 & 72 & 74 & 73 & 72 & 84 \\
Post train & $75^{*}$ & $73^{*}$ & 59 & 57 & $83^{*}$ & $79^{*}$ & 82 \\
\hline
\end{tabular}

Interpretation and Analysis) in mammography. The retrospective trial showed the radiographers recalled all 59 cancers and also called 32 of 90 interval cancers. The radiographers recall rate was 3.9\% higher than the radiologists. ${ }^{12}$ The radiographers then became prospective second readers for 54,000 screening mammograms and there was no significant difference between the radiologist readers and these radiographers in the recall rate $(4 \%)$ or cancer detection rate $(84 \%) .{ }^{12}$

\section{Aim}

This training package was designed to provide radiographers with a systematic method of approaching mammogram assessment as there are no specific breast image interpretation courses available in Australia. While information is available from other sources, this package was designed in a PowerPoint format to provide one easy-to-use resource for busy radiographers. The package is a self-paced tutorial that radiographers can use at any time.

\section{Methods}

Ethics approval for the retrospective study and the inclusion of a training package was provided by the University of Newcastle Human Research Ethics Committee (HREC) in February 2007 (H-352-1206). The assessment methods of two highly respected radiologists in the mammographic field, Drs Lazlo Tabar and Daniel Kopans, ${ }^{13-15}$ were used as the basic framework for the training package. The package provides a disciplined 5-step approach to assessment (Table 1), and each step is expanded with further methods of critique and examples of both the lesions and the results where relevant. The expanded guidelines include a combination of the experience of the researcher and experienced
Table 2: Identification of lesions.

\begin{tabular}{ll} 
Lesion identification \\
\hline 1 & Circumscribed \\
2 & Stellate \\
3 & Lesion, not otherwise specified \\
4 & Asymmetry/distortion \\
5 & Calcifications - granular/casting \\
6 & Calcifications - lobular \\
7 & Calcifications - other NOS \\
\hline
\end{tabular}

radiologists. The package includes basic viewing conditions and techniques, examples of normal anatomical structures, perception issues and areas of the breast that require special attention. Localisation of a lesion using the quadrants of the breast is discussed with in-depth descriptions of lesions, both benign and malignant. The lesions are characterised into seven separate groups and digital images of each lesion type are provided (Table 2).

\section{Participants}

All the radiographers employed at Hunter BreastScreen in 2007 were invited to participate in a pilot study of retrospective image interpretation. Ages ranged from 42 to 62 and experience varied from three years to more than 20 years. This pilot study was conducted for three main reasons; the small number of mammograms to be assessed (50) would enable the radiographers to decide whether they really did want to attempt image interpretation, the selection of the pilot mammograms would provide them with a wide variation of abnormalities and lastly, piloting allowed for fine-tuning of the resource before commencing a larger trial.

\section{Development of the training package}

Identification of abnormalities on mammographic images usually depends on three factors; the quality of the produced images, perception of a lesion and the ability to recognise the significance of that abnormality. ${ }^{16}$ If a lesion is overlooked, a breast cancer will be undetected and therefore 'perception' is the first step in screening assessment. It is critical to develop a systematic method of focusing on small areas of the breast to ensure the entire breast tissue is scrutinised. ${ }^{14-16}$ Once a lesion is detected, it may be considered inconsequential because of a lack of recognition or understanding. ${ }^{7,17,18}$ The training package has been designed to familiarise the radiographers with a variety of common findings and to help enable them to distinguish between normal and abnormal lesions. This should help to increase both their sensitivity (True Positives) and specificity (True Negatives). ${ }^{19,20}$

The training package consists of slides in PowerPoint format and includes teaching aids such as diagrams and images of lesions to demonstrate each category of assessment, and a synopsis of viewing protocols taken from standard text.

Mammographic images of different lesions were collected and scanned using a Vidar Twain 32 (version 4.2) VXR Scanner (Vidar System Corpration, Herndon, VA, USA). The scanning software was opened through Adobe Photoshop 9 (Adobe Systems, San Jose, CA, USA) software. When the films were loaded into the scanner, the scanner gave a low resolution preview. The region of interest (lesions only) was highlighted by drawing a frame around it, the resolution was increased if necessary (the available range was 75 to 300 ) and the scanned images 


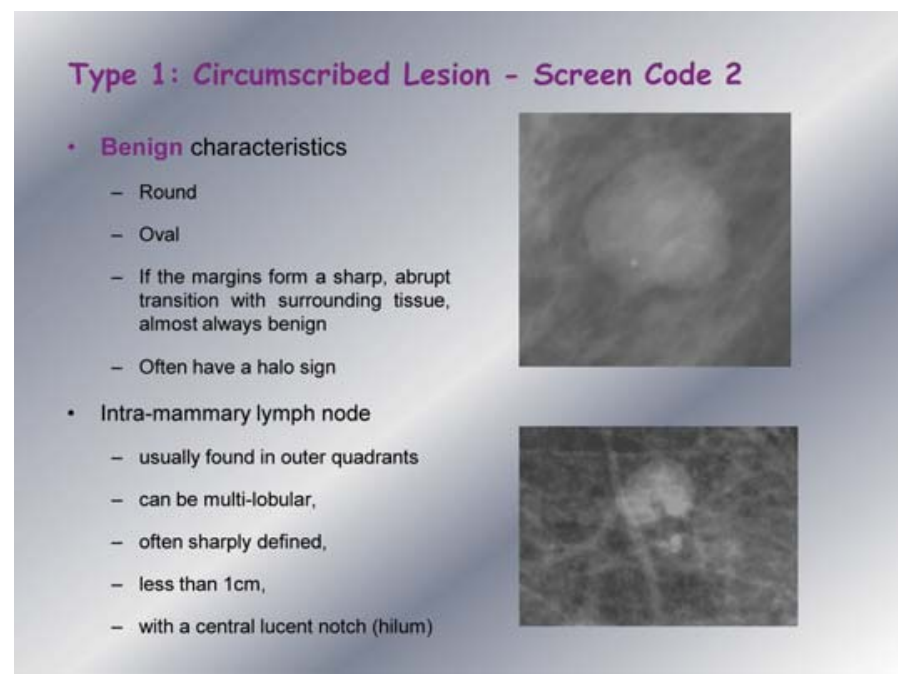

Figure 1: Benign circumscribed lesions.

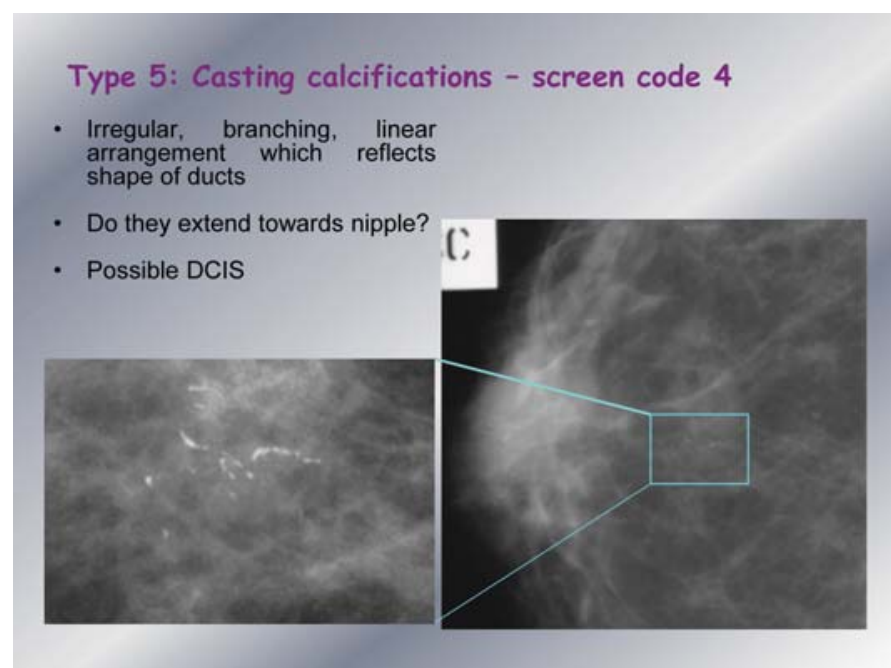

Figure 3: Calcifications - probably malignant.

were downloaded to Photoshop where they were edited. Contrast and brightness of the lesions were manipulated to visually enhance the area of interest. The edited images were saved as a TIFF file, and were copied into a PowerPoint programme. Some examples of the PowerPoint entries as displayed in the training package are demonstrated in Figures 1,2 and 3.

\section{Assessment of the effectiveness of training package}

Eleven radiographers participated in the pilot study ${ }^{21}$ that involved the interpretation of 50 mammograms. After completion of the pilot, all the radiographers were invited to an open-forum informal PowerPoint presentation of the training package. This package could then be accessed on the work computers by all the radiographers at any time and all radiographers were given the opportunity for one-on-one sessions. The larger retrospective study ${ }^{22}$ (250 mammograms) included re-assessment of the 50 mammograms from the pilot study. Seven radiographers completed this study with sufficient numbers to measure the pre- and post-training effects. The pilot study was undertaken in September

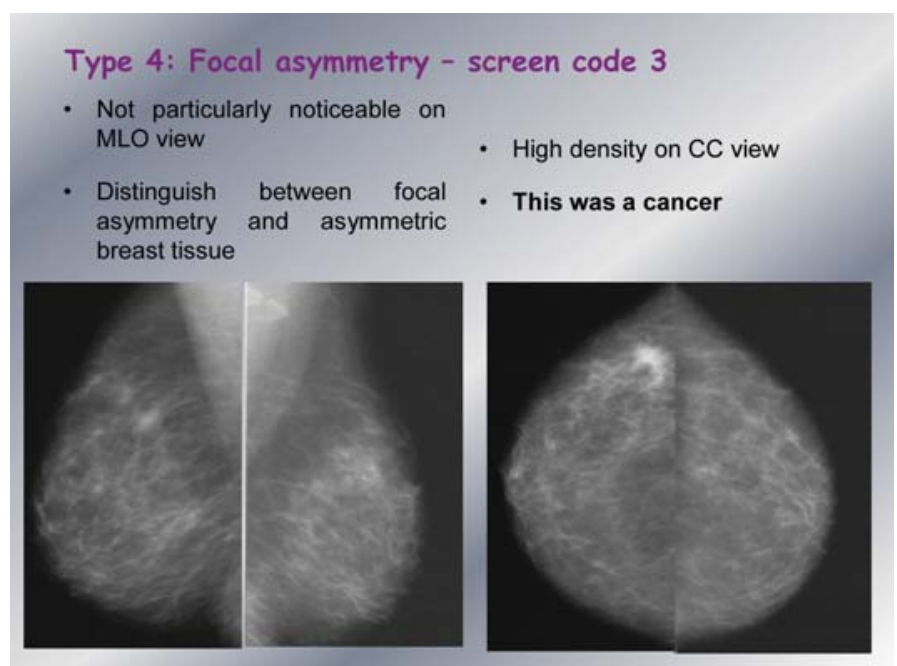

Figure 2: Asymmetry - equivocal at screening.

2007 and these mammograms were re-inserted into the retrospective study between February and October 2008, so although a possibility, it is highly unlikely that memory played any part in the re-assessment. The radiographers were provided with feedback at the conclusion of the retrospective study in the form of their pre- and post-scores. As the prospective study commences, the package will again be offered to the radiographers who wish to refresh their memories.

\section{Results}

When assessing the pilot films pre and post training there was an increase in sensitivity for five radiographers, with three of the same radiographers improving their specificity (Table 3). Overall accuracy improved for four radiographers with a fifth radiographer (R7) maintaining a similar level throughout. However, due to the small sample size these differences were shown to be non-significant (a type II error). It is difficult to say why two radiographers' accuracy decreased so much but it could be due to the fact that the training caused them to "overthink" and, in trying to increase their sensitivity they have recalled more false positives.

\section{Discussion}

Radiographers who were not involved in the study also attended the presentation of the training package; they commented that they found the package interesting and useful for general knowledge. All the radiographers indicated that the package was useful for providing a systematic method with which to commence image interpretation. The self-paced tutorial takes approximately 45-60 minutes and this was considered long enough; if any more detail were required it would be desirable to break the package up into subject modules.

There have been numerous changes in the field of breast imaging since the development of this training package; digital equipment and soft-copy reporting have been widely accepted and installed in many BreastScreen programmes. These changes have necessitated some alteration to the training package, mostly with regards to the preparation for batch reading of mammograms. No longer will masking of extraneous light on viewboxes be necessary, and the lack of viewboxes will enhance the ambient light in digital workplaces. Digital mammography has made 
the method of physically masking images impractical, ${ }^{16}$ but the principle remains the same.

Soft-copy reporting monitors allow the reader to visualise details in dense breast tissue and in the sub-dermal layers simultaneously and provides for manipulation of suspect or dense areas. ${ }^{23}$ Often the reader must pan through the image to view all the information recorded, and this has proved to be more time consuming than reading hard copy images. ${ }^{23,24}$ The training package will be modified to provide information for radiographers on optimal techniques for image processing and manipulation. Lesions in the breasts will remain constant. They may be easier to perceive with the high-resolution monitors. During the retrospective study, the radiographers had most difficulty with perception of circumscribed lesions and calcifications. ${ }^{22}$ The training package will be modified to target those lesions and provide more information on how to determine their significance.

The ranges of sensitivity and specificity for radiographers following this training package are comparable with those reported by Van den Biggelaar, et al..$^{10}$ Three studies in the review had pre and post-training performance measurements, demonstrating that radiographers in only one study increased in both sensitivity $(77-83 \%)$ and specificity (68$80 \%$ ). While the improvements noted in this study were not statistically significant, this may be due to the relatively small numbers of images assessed.

\section{Conclusion}

Any extra training in a modality enhances the participants' knowledge on that particular subject and may show improvement immediately after the training ${ }^{25-27}$ but unless these new skills are used continuously they are likely to abate with time. In Australia there is no formal training available for mammographic image interpretation, so this training package has been developed simply to allow radiographers who are interested, to increase their knowledge and update their skills in this field until some formal education becomes a reality.

\section{References}

1 AIHW. Breast Cancer in Australia: An overview, 2009. Canberra, 2009

2 Royal Australian New Zealand College of Radiologists. Work Survey, RANZCR 2006.

3 Bassett LW, Monsees BS, Smith RA, Wang L, Hooshi P, Farria DM, et al. Survey of Radiology Residents: Breast Imaging Training and Attitudes. Radiology 2003; 227: 862-9.

4 Thompson W, Pollard K. The current status of radiographers as screen readers in breast screening units: applications for Australia. The Radiographer 2007; 54: 16-20.

5 BreastScreen Australia. National Accreditation Standards (NAS). 2008.

6 Duijm L, Groenewoud J, Fracheboud J, van Ineveld BM, Roumen R, de Koning $\mathrm{HJ}$. Introduction of additional double reading of mammograms by radiographers: Effects on a biennial screening programme outcome. Eur J Cancer 2008; 44 1223-8.

7 Elmore JG, Brenner J. The More Eyes, the Better to See? From double to quadruple reading of screening mammograms. J Natl Cancer Inst 2007: 1141-3.

8 Brewer NT, Salz T, Lillie SE. Systematic review: The long-term effects of falsepositive mammograms. Ann Intern Med 2007; 146: 502-10.
9 Duijm LEM, Groenewoud JH, Fracheboud J, de Koning HJ. Additional double reading of screening mammograms by radiologic technologists: impact on screening performance parameters. J Natl Cancer Inst 2007; 99: 1162-70.

10 Van den Biggelaar F, Nelemans P, Flobbe K. Performance of radiographers in mammogram interpretation: a systematic review. The Breast 2008; 17: 87-92.

11 Van den Biggelaar F, Nelemans P, Flobbe K. Performance of radiographers in mammogram interpretation: a systematic review. The Breast 2007; 17: 87-92.

12 Wivell G, Denton ERE, Eve CB, Inglis JC, Harvey I. Can radiographers read screening mammograms? Clin Radiol 2003; 58: 63-7.

13 Tabar L, Tot T, Dean P. Breast Cancer: The art and science of early detection with mammography. New York: Thieme; 2005.

14 Kopans DB. Breast Imaging Third edition. Philadelphia: Lippincott Williams \& Wilkins; 2007.

15 Tabar L. Teaching course in diagnostic breast imaging: multi-modality approach to the detection and diagnosis of occult breast cancer. Cave Creek: Mammography Education Inc.; 2007.

16 Hashimoto BE. Multimodality breast imaging; a correlative atlas Second edition. New York: Thieme Medical Publishers; 2010.

17 Bird RE, Wallace TW, Yankaskas BC. Analysis of cancers missed at screening mammography. Radiology 1992; 184: 613-7.

18 Yankaskas BC, Schell MJ, Bird RE, Desrochers DA. Reassessment of breast cancers missed during routine screening mammography: a community-based study. $A J R$ Am J Roentgenol 2001; 177: 535-41.

19 Warner S. Truth Tables: Effectively evaluate new tests and how they will perform in patient populations. Available onilne at www.Advanceweb.com [verified 14th June 2010].

20 Spitalnic S. Test properties 1: sensitivity, specificity, and predictive values. Hospital Physician 2004: 27-31.

21 Moran S, Warren-Forward H. A retrospective pilot study of the performance of mammographers in interpreting screening mammograms. The Radiographer 2010; 57: 12-9.

22 Moran S, Warren-Forward H. A comparative analysis of screening mammography review between mammographers and gold standard screen readers in a retrospective study. J Med Imaging Radiat Oncol 2009; 53: A247.

23 Nishikawa RM, Acharyya S, Gatsonis C, Pisano ED, Cole EB, Marques HS, et al. Comparison of soft-copy and hard-copy reading for full-field digital mammography. Radiology 2009; 251: 41-9.

24 Haygood TM, Whitman GJ, Atkinson EN, Nikolova RG, Sandoval SYC, Dempsey PJ. Results of a survey on digital screening mammography: prevalence, efficiency and use of ancillary diagnostic aids. J Am Coll Radiol 2008; 5: 585-92.

25 Mackay SJ. The impact of a short course of study on the performance of radiographers when highlighting fractures on trauma radiographs: "The Red Dot System". Br J Radiol 2006; 79: 468-72.

26 Linver MN, Paster SB, Rosenberg RD, Key CR, Stidley CA, King WV. Improvement in mammography Interpretation skills in a community radiology practice after dedicated teaching courses: 2-year medical audit of 38,633 cases. Radiology 1992; 184: $39-43$.

27 Ciatto S, Ambrogetti D, Morrone D, Rosselli Del Turco M. Analysis of the results of a proficiency test in screening mammography at the CSPO of Florence: Review of 705 tests. Radiol Med 2007; 111: 797-803.

28 Haycock A, Burling D, Wylie P, Muckian J, Ilangovan R, Thomas-Gibson S. CT colonography training for radiographers - a formal evaluation. Clin Radiol 2010; 65: 997-1004. 